\title{
MODULARITY* IN LIE ALGEBRAS
}

\author{
by K. BOWMAN and V. R. VAREA
}

(Received 1st February 1995)

\begin{abstract}
A subalgebra $U$ of a Lie algebra $L$ over a field $F$ is called modular* in $L$ if $U$ satisfies the dual of the modular identities in the lattice of subalgebras of $L$. Our aim is the study of the influence of the modular* identities in the structure of the algebra. First we prove that if the modular* conditions are imposed on an ideal of $L$ then every element of $L$ acts as an scalar on this ideal and if they are imposed on a non-ideal subalgebra $U$ of $L$ then the largest ideal of $L$ contained in $U$ also satisfies the modular* identities. We determine Lie algebras having a subalgebra which satisfies both the modular and modular* identities, provided that either $L$ is solvable or $\operatorname{char}(F) \neq 2,3$. As immediate consequences of this result we obtain that the existence of a coatom satisfying the modular* identities in the lattice $\mathcal{C}(L)$ forces that the lattice $\mathcal{L}(L)$ is modular and that the modular* identities on any subalgebra $U$ forces that $U$ is quasi-abelian. In the case when $L$ is supersolvable we obtain that the modular* conditions on any non-ideal of $L$ are enough to guarantee that $\mathcal{L}(L)$ is modular. For arbitrary fields and any Lie algebra $L$, we prove that the modular* conditions on every co-atom of the lattice $\mathcal{L}(L)$ guarantee that $\mathcal{L}(L)$ is modular.
\end{abstract}

1991 Mathematics subject classification: 17B05.

\section{Introduction}

Throughout $L$ will denote a finite dimensional Lie algebra over a field $F$. We say that $L$ is almost-abelian if $L$ has a basis $a_{1}, \ldots, a_{n}, x$ with product given by $\left[a_{i}, a_{j}\right]=0$ and $\left[a_{i}, x\right]=a_{i}$ for every $i, j$. If $L$ is either abelian or almost-abelian we say that $L$ is quasi-abelian. The core of a subalgebra $S$ of $L$, denoted by $S_{L}$, is the largest ideal of $L$ contained in $S$. If $S_{L}=0$ we say $S$ is core-free in $L$.

A subalgebra $U$ of $L$ is called modular in $L$ if it is a modular element in the lattice of subalgebras of $L$; that is

$$
\langle U, B\rangle \cap C=\langle B, U \cap C\rangle \text { for all subalgebras } B \leq C
$$

and

$$
\langle U, B\rangle \cap C=\langle B \cap C, U\rangle \text { for all subalgebras } U \leq C .
$$

Here $\langle X, Y\rangle$ denotes the subalgebra of $L$ generated by $X$ and $Y$. (The easiest example of modular subalgebras are the subalgebras $Q$ of $L$ such that $[Q, V] \subseteq Q+V$ for every subspace $V$ of $L$, such subalgebras are called quasi-ideals). Quasi-ideals have been studied in [1], [5] and [15]. The only known examples of modular subalgebras which 
are not quasi-ideals are the one dimensional subalgebras of the three dimensional non-split simple Lie algebras and the standard maximal subalgebra of the hamiltonian Lie algebra $\mathcal{H}(2: \underline{1}: \Phi(\gamma))^{2}$ (see [16]).

Although many properties of modular subalgebras have been obtained (see $[2,6,7$, $13,14,16])$ the general problem of determining the Lie algebras with modular subalgebras is still open. One of the known results is that every modular subalgebra $U$ of $L$ is a quasi-ideal of $L$, except when $L$ is three dimensional non-split simple, provided that either $L$ is solvable or $\operatorname{char}(F)=0$ or $L$ is restricted and $F$ is algebraically closed of characteristic $p>7$ (see [16]). So, under the cited conditions, if $U$ is core-free and modular in $L$ then $L$ is either almost-abelian, three-dimensional non-split simple or a Zassenhaus algebra (see [1]). For perfect fields of characteristic different from two or three, it is also known that if a minimal subalgebra $A$ of $L$ is modular then either $A \triangleleft L$ or $L$ is either almost-abelian or three dimensional non-split simple (see [13]) and that if a rank one simple subalgebra of $L$ is modular then it must be an ideal of $L$ (see [15]).

The modular condition can be dualised to give, a subalgebra $U$ of $L$ is called modular* in $L$ if

$$
\langle U, B\rangle \cap C=\langle B, U \cap C\rangle \text { for all subalgebras } B \leq C
$$

and

$$
\langle U \cap B, C\rangle=\langle B, C\rangle \cap U \text { for all subalgebras } C \leq U .
$$

Modular* subalgebras have been introduced in [3]. Note that if $U$ is a maximal subalgebra of $L$ and modular* in $L$ then $U$ is modular in $L$. Dually, every minimal subalgebra of $L$ which is modular in $L$ is modular*.

The easiest example of subalgebras satisfying the modular* identities are the subalgebras $U$ such that $\langle u, S\rangle=((u))+S$ for every subalgebra $S$ of $L$ and $u \in L$, where $((u))$ denotes the subspace of $L$ generated by $u$; in other words, the subalgebras $U$ such that $((u))$ is a quasi-ideal of $L$ for every $u \in U$ (these subalgebras will be called strong quasi-ideals). We will say that an ideal $N$ of $L$ is a strong ideal if $((a))$ is an ideal of $L$ for every $a \in N$. We have the following chains of implications:

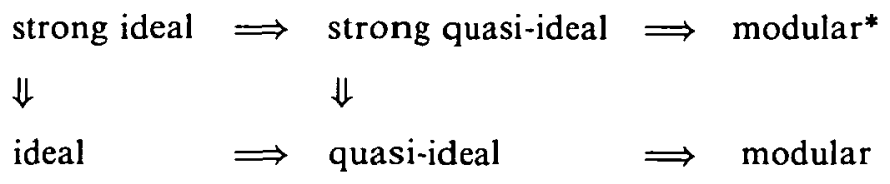

In this paper, first we characterize quasi-ideals which are modular* and cores of modular* subalgebras. We show that if a quasi-ideal is modular* in $L$ then either it is a strong ideal or $L$ is almost-abelian. We prove that the core of a modular* subalgebra of $L$ is also modular* and it is a strong ideal. We are able to characterize Lie algebras having subalgebras which are both modular and modular*, in the cases when either 
$\operatorname{char}(F) \neq 2,3$ or $L$ is solvable. Then, as maximal and modular* subalgebras are modular, Lie algebras having a maximal and modular* subalgebra will be determined. After that, we will be able to obtain that every modular* subalgebra must be quasiabelian, in those cases. In the general case, we obtain that the modular* conditions on every maximal subalgebra of $L$ force that the $\mathcal{L}(L)$ is modular. Some similar questions have been looked at by Towers in [11], however the approach is quite different.

It is known that for fields of characteristic different from two or three, the lattice $\mathcal{L}(L)$ is modular if and only if $L$ is either quasi-abelian or a $\mu$-algebra (this means that every proper subalgebra of $L$ is one dimensional), see [9] or [18, Corollary 5]. If $F$ is perfect and $\operatorname{char}(F) \neq 2,3$ then every $\mu$-algebra is three dimensional non-split simple (see [6, Proposition 1]).

Finally in this section we give two easy lemmas that will be useful throughout this paper.

Lemma 1.1. If $N$ is a proper subalgebra of $L$ for which $[n, x] \in((n))$ for some $n \in N$ and all $x \in L-N$, then $((n))$ is an ideal of $L$.

Proof. Let $m \in N$. Then $m+x \notin N$, so $[n, m+x] \in((n))$, whence $[n, m] \in((n))$.

Lemma 1.2. If $Q$ is a proper subalgebra of $L$ for which $[q, x] \in((q))+((x))$ for some $q \in Q$ and all $x \in L-Q$, then $((q))$ is a quasi-ideal of $L$.

Proof. Let $r \in Q$. Then $r+x \notin Q$, so $[q, r+x] \in((q))+((r+x))$, whence $[q, r] \in$ $\{((q))+((r))+((x))\} \cap Q=((q))+((r))$.

\section{Quasi-ideals and modular* subalgebras}

We begin studying relationships between quasi-ideals and modular* subalgebras. Examples of modular* which are not strong quasi-ideals are the following: (i) every proper subalgebra of a three dimensional non-split simple Lie algebra, (ii) every one dimensional subalgebra of $L$ contained in $A$, being $L=A+((x))$ where $A$ is an abelian minimal ideal of $L$. We note that the subalgebras cited in (i) and (ii) above are not quasi-ideals.

Lemma 2.1. An ideal $N$ of a Lie algebra $L$ is a strong ideal if and only if $N$ is abelian and each element of $L$ acts as a scalar on $N$.

Proof. Suppose that $N$ is a strong ideal. We may assume $\operatorname{dim} N>1$. Let $x, x^{\prime}$ be linearly independent elements of $N$. We see that $\left[x, x^{\prime}\right] \in((x)) \cap\left(\left(x^{\prime}\right)\right)=0$. This yields that $N$ is abelian. Moreover, for each element $y \in L-N$, we have $[x, y]=\lambda x, x^{\prime} y=\mu x^{\prime}$ and $\left[x+x^{\prime}, y\right]=\alpha\left(x+x^{\prime}\right)$, where $\lambda, \mu, \alpha \in F$. This yields that $\lambda=\mu$. Therefore each element of $L-N$ acts a scalar on $N$. The converse is clear. 
Lemma 2.2. If $Q$ is a strong quasi-ideal of $L$, then either $Q$ is a strong ideal or $L$ is almost-abelian.

Proof. Assume $Q$ is a strong quasi-ideal and that there exists $q \in Q$ such that $((q))$ is not an ideal of $L$. Then, from Theorem 3.6 of [1] it follows that $L$ is almost-abelian. Then assume $((q)) \triangleleft L$ for every $q \in Q$. Clearly, $Q$ is an abelian ideal of $L$. Hence $Q$ is a strong ideal of $L$.

Proposition 2.3. Let $Q$ be a proper quasi-ideal of a Lie algebra $L$ which is modular* in $L$. Then, $Q$ is a strong quasi-ideal and so either $Q$ is a strong ideal or $L$ is almostabelian.

Proof. Assume $Q$ is modular*. Let $q \in Q$ and $x \in L-Q$. We have $[q, x] \in[Q, x]=$ $Q+((x))$. So, $[q, x]-\lambda(q, x) x \in Q$ for some $\lambda(q, x) \in F$. Put $C=((q))$ and $B=((x))$. We have $\langle Q \cap B, C\rangle=C=((q))$, and $[q, x]-\lambda(q, x) x \in\langle B, C) \cap Q$. Now, by using the second modular* identity we obtain $q^{\prime} \in((q))$ thus $[q, x] \in((q))+((x))$. Thus $((q))$ is a quasi-ideal of $L$ for every $q \in Q$ by Lemma 1.2. Hence, $Q$ is a strong quasi-ideal. The last assertion in the proposition follows from Lemma 2.2 .

Proposition 2.4. Let $U$ be a proper modular* subalgebra of L. Then the following holds:

(i) $U_{L}$ is a strong ideal of $L$.

(ii) $U_{L}=\{u \in U \mid((u)) \unlhd L\}$.

Proof. (i) Let $u \in U_{L}$ and $x \in L-U$. Put $C=((u))$ and $B=((x))$. From the second modular* identity we have $((u))=C=\langle B, C\rangle \cap U=\langle u, x\rangle \cap U$. As $U_{L}$ is an ideal of $L$, we have $[u, x] \in U_{L} \cap\langle u, x\rangle \cap U$. Therefore, $[u, x] \in((u))$. Therefore by Lemma 1.1, $((u)) \unlhd L$ for every $u \in U_{L}$. So that $U_{L}$ is a strong ideal of $L$.

(ii) Write $K=\{u \in U \mid((u)) \unlhd L\}$. From (i) it follows $U_{L} \leq K$. Let $u, v \in K$. Clearly, $[u, v]=0$ and $\lambda u \in K$ for every $\lambda \in F$. Now we prove that $u+v \in K$. To do that take $x \in L-K$. By the second modular* identity, we find $\langle u+v, x\rangle \cap U=((u+v))$. Since, $[u+v, x] \in U \cap\langle u+v, x\rangle$, we get $[u+v, x] \in((u+v))$. So that $((u+v)) \unlhd L$. Therefore, $K$ is an ideal of $L$. Thus $K \leq U_{L}$. This completes the proof.

We say that an ideal $N$ of $L$ is supersolvably immersed in $L$ if every chief factor of $L$ below $N$ is one dimensional.

Corollary 2.5. Let $U$ be a proper modular* subalgebra of L. Then the following holds:

(i) $U_{L}+((y))$ is quasi-abelian for every $y \in L$.

(ii) $U_{L}$ is supersolvably immersed in $L$. 


\section{The solvable case}

In this section we determine modular and modular* subalgebras in solvable Lie algebras $L$ over any field. First we consider the case when $L$ is supersolvable. In this case we obtain that if a non-ideal of $L$ is modular* then $L$ is almost-abelian. Then, by using results of the previous section we will be able to prove that if a solvable Lie algebra $L$ has a non-ideal, modular and modular* subalgebra then it is almost-abelian. From this it is easy to obtain that if a maximal subalgebra of $L$ satisfies the modular* identities then $L$ is almost-abelian and that every modular* subalgebra of any solvable Lie algebra is quasi-abelian. The results in this section will be used in the next section.

We begin with the following

Lemma 3.1. Let $L$ be any Lie algebra and let $U$ be modular* in $L$. Then for every $x \in L-U$ and $0 \neq u \in U$, the subalgebra $((x))$ is maximal in $\langle u, x\rangle$.

Proof. Let $M$ be a maximal subalgebra of $\langle u, x\rangle$ containing $((x))$. By using the second modular* identity, we obtain

$$
((u))=\langle U \cap((x)), u\rangle=\langle u, x\rangle \cap U .
$$

This yields, $U \cap M \leq U \cap\langle u, x\rangle=((x))$. Since $M \neq\langle u, x\rangle$, it follows $u \notin M$. Hence, $U \cap M=0$. Now, by using the first modular* identity, we obtain

$$
\langle U, x\rangle \cap M=M=\langle x, U \cap M\rangle=((x)) .
$$

Hence, $((x))$ is maximal in $\langle u, x\rangle$.

Theorem 3.2. Let $L$ be supersolvable over any field and let $U$ be a proper modular* subalgebra of $L$. Then, either $U$ is a strong ideal of $L$ or $L$ is almost-abelian.

Proof. We want to prove that $U$ is a quasi-ideal of $L$. First take $u \in U$ and $x \in L-U$. By Lemma 3.1, ((x)) is maximal in $\langle u, x\rangle$. As $\langle u, x\rangle$ is supersolvable, we have that $((x))$ has codimension one in $\langle u, x\rangle$. Therefore $[u, x] \in((u))+((x))$. Therefore $U$ is a quasi-ideal of $L$ by Lemma 1.2. Then the result follows from Proposition 2.3.

Now we can determine the solvable Lie algebras having a modular and modular* subalgebra.

Theorem 3.3. Let $L$ be solvable over any field $F$. Let $U$ be a proper subalgebra of $L$ which is modular and modular* in $L$. Then either $U$ is a strong ideal or $L$ is almostabelian.

Proof. If $U \triangleleft L$, then $U$ is a strong ideal by Proposition 2.4. Suppose then $U$ is not an ideal of $L$. Since $U$ is modular in $L$, by Corollary 1.2 of [14] it follows that 
$L / U_{L}$ is almost-abelian. As $U$ is modular* in $L$, by Corollary 2.5 (ii) it follows that $U_{L}$ is supersolvably immersed in $L$. Then we have that every chief factor of $L$ has dimension one. So, $L$ is supersolvable. Therefore, by Theorem 3.2 it follows that $L$ is almost-abelian.

Next we determine the solvable Lie algebras having a maximal subalgebra which is modular*.

Corollary 3.4. Let $L$ be solvable over any field. Then the following are equivalent:

(i) L has a maximal subalgebra which is modular* in $L$.

(ii) The lattice $\mathcal{L}(L)$ of all subalgebras of $L$ is modular.

(iii) L is quasi-abelian.

Proof. (i) implies (iii): Suppose that $M$ is maximal and modular* in $L$. Then, we have that $M$ is also modular in $L$. Therefore, by Theorem 3.3, either $M$ is a strong ideal or $L$ is almost-abelian. In the former case, we have $\operatorname{dim} L / M=1$ and by Corollary 2.5 it follows that $L$ must be quasi-abelian. Clearly, (iii) implies (ii). If $\mathcal{L}(L)$ is modular, then every subalgebra of $L$ is modular and modular* in $L$. So, (ii) implies (i). The proof is complete.

As an immediate consequence of the above corollary we have

Corollary 3.5. Let $L$ be solvable over any field. Then every proper subalgebra of $L$ which is modular* in $L$ is quasi-abelian.

Proof. Suppose that $U<L$ is modular* in $L$. Take a subalgebra $M$ of $L$ containing $U$ such that $U$ is maximal in $M$. Then, by Corollary $3.4, M$ is quasi-abelian. Therefore, $U$ is quasi-abelian too.

\section{The non-solvable case}

In this section we consider the case when the Lie algebra $L$ is nonsolvable over a field $F$ with $\operatorname{char}(F) \neq 2,3$. We obtain that if $L$ has a non-ideal, modular and modular* subalgebra then every proper subalgebra of $L$ is one dimensional (such a Lie algebra is called a $\mu$-algebra). Then, by using results of the previous sections, it is easy to prove that if $L$ has a maximal subalgebra satisfying the modular* identities then $L$ must be a $\mu$-algebra and that every modular* subalgebra of any Lie algebra is quasi-abelian.

The proof of the main result depends heavily on results on supersimple Lie algebras which appear in [12] and [18]. A Lie algebra $L$ is said to be supersimple if every subalgebra of $L$ of dimension greater than one is simple. Every $\mu$-algebra is supersimple. More generally, a Lie algebra of dimension greater than one is supersimple if and only if it has no two dimensional subalgebras (Proposition 3.2 of [12]). If $\operatorname{char}(F) \neq 2,3$ and if $L$ is supersimple, then for each $0 \neq x \in L$ there exists 
$y \in L$ such that $L=\langle x, y\rangle$ (Theorem 4 of [18]). We recall that for perfect fields of characteristic different from two or three, the only supersimple Lie algebras are the three dimensional non-split simple (it follows from Proposition 1 of [6]).

First we consider core-free, modular and modular* subalgebras of a nonsolvable Lie algebra $L$. More generally, we give the following

Lemma 4.1. Let $L$ be a Lie algebra over an arbitrary field $F$. Let $U$ be a core-free subalgebra of $L$ such that $\langle u, z\rangle$ is either two dimensional or a $\mu$-algebra for every $0 \neq u \in U$ and $z \in L-U$. Then one of the following holds:

(i) $L$ is almost-abelian.

(ii) $\langle u, z\rangle$ is a $\mu$-algebra for every $0 \neq u \in U$ and $z \in L-U$.

Proof. Pick $0 \neq u \in U$. Suppose that $\langle u, z\rangle$ is two dimensional for every $z \in L-U$. Then $[u, z] \in((u))+((z))$. Hence $((u))$ is a quasi-ideal of $L$ by Lemma 1.2. As $U$ is corefree, $((u))$ is not an ideal of $L$. So, by [1] it follows that $L$ is almost-abelian. Now suppose that there exist $x, z \in L-U$ such that $\langle x, u\rangle$ is two dimensional and $\langle u, z\rangle$ is a $\mu$-algebra. Write $Q=\langle x, u\rangle$ and $T=\langle u, z\rangle$. We have $Q \cap T=((u))$. Let us first suppose $((u)) \triangleleft Q$. Then $[x, u]=\alpha u$ where $\alpha \in F$. Since $[x+z, u]=\alpha u+[z, u]$ and since $[x+z, u] \in\langle x+z, u\rangle$, we have $[z, u] \in\langle x+z, u\rangle$. So, $\langle u,[z, u]\rangle=T \leq\langle x+z, u\rangle$. This yields that $\langle x+z, u\rangle$ has dimension greater than two. Then, $\langle x+z, u\rangle$ must be a $\mu$-algebra. Therefore, $T=\langle x+z, u\rangle$. But then we have $x \in T$ and so $Q \leq T$, which is a contradiction. Now suppose that $((u))$ is not an ideal of $Q$. Let $q \in Q$ such that $((q)) \triangleleft Q$. Then $[q, u]=\beta q$ where $\beta \in F$ and $((u)) \neq((q))$. We have $[q+z, u]=\beta q+[z, u] \in\langle q+z, u\rangle$. This yields

$$
-\beta z-[u, z]=-\beta z+[z, u]=\beta q+[z, u]-\beta(q+z) \in\langle q+z, u\rangle .
$$

So that, $0 \neq \beta z+[u, z] \in\langle q+z, u\rangle$. Since $\beta z+[u, z] \notin((u))$, we have $T=\langle\beta z+[u, z], u\rangle \leq$ $\langle q+z, u\rangle$. Therefore, $\langle q+z, u\rangle$ has dimension greater than 2 . It follows that $\langle q+z, u\rangle$ must be a $\mu$-algebra. Hence $T=\langle q+z, u\rangle$. This yields, $q \in T \cap Q=((u))$, which is a contradiction. We deduce that either $L$ is almost-abelian or $\langle u, z\rangle$ is a $\mu$-algebra for every $0 \neq u \in U$ and $z \in L-U$.

Proposition 4.2. Let $F$ be any field of characteristic different from two or three and let $L$ be a non-solvable Lie algebra. Assume that $U$ is a proper core-free, modular and modular* subalgebra of $L$. Then $L$ is a $\mu$-algebra.

Proof. Let us first suppose $\operatorname{dim} U=1$. Write $U=((u))$. In the case when $F$ is perfect, then Theorem 2.2 of [13] applies and $L$ is three dimensional non-split. In the case when $F$ is not perfect we need to make a slight variation of the proof of that theorem by using results of [17] and [18]. In the proof of Theorem 2.2 of [13] it is proved that $L$ has no two dimensional abelian subalgebras. Now we prove that $L$ has no two dimensional nonabelian subalgebras either. Before that we claim that $U$ satisfies the conditions in Lemma 4.1. Let $0 \neq u \in U$ and $z \in L-U$. Since $U$ is 
modular in $L$ we have that $U$ is maximal and modular in $\langle u, z\rangle$ (see [2]). Then we get that $\langle u, z\rangle$ is either two dimensional or a $\mu$-subalgebra (see the proof of Theorem 2.2 of [13]). Thus Lemma 4.1 applies and $\langle u, z\rangle$ is a $\mu$-algebra for every $0 \neq u \in U$ and $z \in L-U$. Suppose that $S$ is a two dimensional nonabelian subalgebra of $L$. Let $x \in S$ such that $((x)) \triangleleft S$. Since $U$ is self-normalizing, $U \neq((x))$. Then $\langle u, x\rangle$ is a $\mu$-algebra and $U<\langle u, x\rangle<\langle u, S\rangle$. We can suppose without loss of generality that $\langle u, S\rangle=L$. Let $0 \neq N$ be an ideal of $L$. Pick $0 \neq y \in N$. We have that $\langle U, y\rangle \cap N$ is a nonzero ideal of $\langle U, y\rangle$. Since $\langle U, y\rangle$ is simple, it follows $U \leq N$. But since $U$ is self-normalizing in $L$ (see [2]), we get $N=L$. Therefore, $L$ is simple. By the modularity of $U$ it follows that $S$ is maximal in $L$. Since $\langle u, x\rangle$ is a $\mu$-algebra we have that $x$ is not ad-nilpotent. This yields that $S$ coincides with the Fitting null component of ad $x$, which contradicts Proposition 1.9 of [17]. Therefore $L$ has no two dimensional subalgebras and hence $L$ is supersimple (Proposition 3.2 of [12]). As $\operatorname{char}(F) \neq 2,3$, by Theorem 4 of [18] there exists $z \in L$ such that $L=\langle u, z\rangle$. We conclude that $L$ is a $\mu$-algebra.

Next we prove that $U$ must be one dimensional. Assume $\operatorname{dim} U>1$. Let $L$ be a counterexample of minimal dimension. By Proposition 2.3 and Lemma 2.1, $U$ is selfnormalizing. So, by the minimality of $L$ we have that $U$ is a maximal subalgebra of $L$. Now we claim that $U$ is supersimple. To do that we prove that $U$ has no two dimensional subalgebras. Suppose that $S$ is a two dimensional subalgebra of $U$ and let $u, u^{\prime}$ be a basis for $S$ with $\left[u, u^{\prime}\right]=\alpha u$ where $\alpha \in F$. Let $z \in L-U$. By the second modular* identity we have $((u))=U \cap\langle u, z\rangle$. Now from the modularity of $U$ it follows that $((u))$ is modular in $\langle u, z\rangle$. So, by Theorem 2.2 of [13] it follows that $\langle u, z\rangle$ is two dimensional or a $\mu$-algebra. So, by Lemma $4.1,\langle u, z\rangle$ is a $\mu$-algebra for every $0 \neq u \in U$ and $z \in L-U$. On the other hand, we have

$$
\left[u, u^{\prime}+z\right]=\left[u, u^{\prime}\right]+[u, z]=\alpha u+[u, z] \in\left\langle u, u^{\prime}+z\right\rangle
$$

This yields $\langle u,[u, z]\rangle \leq\left\langle u, u^{\prime}+z\right\rangle$. As $\langle u, z\rangle$ and $\left\langle u, u^{\prime}+z\right\rangle$ are both $\mu$-algebras, we have $\langle u, z\rangle=\langle u,[u, z]\rangle=\left\langle u, u^{\prime}+z\right\rangle$. This yields $u^{\prime} \in\langle u, z\rangle \cap U=((u))$, which is a contradiction. Therefore, $U$ has no two dimensional subalgebras. The claim is proved.

For perfect fields of characteristic different from two or three, every supersimple subalgebra is three dimensional non-split simple. So by Theorem 1.5 of [15] we have $U \triangleleft L$ which is a contradiction. For arbitrary fields, we need to work more.

Next we prove that $L$ is also supersimple. Assume not. Then by [12], $L$ has a subalgebra $Q$ of dimension two. Since $U$ is supersimple, $S$ is not contained in $U$. Since $U$ is maximal and modular, we have $U \cap Q \neq 0$. Take $0 \neq u \in U \cap Q$ and $x \in Q, x \notin U$. We have that $\langle u, x\rangle$ is two dimensional. By Lemma 1 , it follows that $L$ is almost-abelian which is a contradiction. Now take any $0 \neq u \in U$. As $\operatorname{char}(F)=2,3$ and $L$ is supersimple, Theorem 4 of [18] applies and there exist an element $v \in L-U$ such that $L=\langle u, v\rangle$. But then we have $\langle U \cap((v)), u\rangle=((u))$ whereas $\langle v, u\rangle \cap U=L$ and so the second modular* identity does not hold for $U$. This contradiction shows that $\operatorname{dim} U=1$ and hence the result.

Theorem 4.3. Let $F$ be any field of characteristic different from two or three. Let $U$ 
be a proper modular and modular* subalgebra of a non-solvable Lie algebra $L$. Then either $U$ is a strong ideal or $L$ is a $\mu$-algebra.

Proof. If $U$ is an ideal of $L$, then $U$ is a strong ideal of $L$ by Proposition 2.4. Then suppose that $U$ is not an ideal of $L$. We have that $U / U_{L}$ is a core-free, modular and modular* subalgebra of $L / U_{L}$. Then, by Proposition 4.2 , it follows that $L / U_{L}$ is a $\mu$-algebra. Assume $U_{L} \neq 0$. By Corollary $2.5, U_{L}$ is abelian and it is supersolvably immersed in $L$. Thus we may suppose without loss of generality that $\operatorname{dim} U_{L}=1$. Put $U_{L}=((a))$. Let $u \in U-((a))$ and $z \in L-U$. By the second modular* identity we have $((u))=U \cap\langle u, z\rangle$. This yields $U_{L} \pm\langle u, z\rangle$. Since $L / U_{L}$ has no proper subalgebras of dimension greater than one, $L=U_{L}+\langle u, z\rangle$. Therefore, $\langle u, z\rangle$ is a $\mu$-algebra for every $u \in U-((a))$ and $z \in L-U$. On the other hand, we have that $u, z$ and $[u, z]$ act as scalars on $U_{L}$ by Proposition 2.4 and Lemma 2.1. Thus $[u, a]=\lambda a,[z, a]=\theta a$ and $[[u, z], a]=\rho a$ where $\lambda, \theta, \rho \in F$. By the Jacobi identity, we have $\rho a=[[u, z], a]=$ $[u,[z, a]]+[[u, a], z]=\theta[u, a]+\lambda[a, z]=0$. Put $y=[u, z]$. We see that $\langle u, z\rangle=\langle u, y\rangle$ and that $\langle a+u, y\rangle$ is a $\mu$-algebra. Moreover we have $[u, y]=[a+u, y] \in\langle y, a+u\rangle$. This yields, $\langle u, y\rangle=\langle y,[y, u]\rangle \leq\langle a+u, y\rangle$ and hence $\langle a+u, y\rangle=\langle u, y\rangle$. So, $a \in\langle u, y\rangle=\langle u, z\rangle$, which is a contradiction. Thus $U_{L}=0$ and $L$ is a $\mu$-algebra. The proof is complete.

Corollary 4.4. Let $F$ be a perfect field of characteristic $\neq 2,3$. Let $U$ be a modular and modular* subalgebra of a non-solvable Lie algebra $L$. Then either $U$ is a strong ideal or $L$ is three dimensional non-split simple.

Now we are able to determine the nonsolvable Lie algebras having a maximal subalgebra which is modular*.

Corollary 4.5. Let $F$ be of characteristic $\neq 2,3$. For a nonsolvable Lie algebra $L$ the following are equivalent:

(i) L has a maximal subalgebra which is modular* in $L$.

(ii) $L$ is a $\mu$-algebra.

Proof. (i) implies (ii): Suppose $M$ is a maximal subalgebra of $L$ which is modular* in $L$. We have that $M$ is also modular in $L$. So, by Theorem 4.3 it follows that either $M$ is a strong ideal, or $L$ is a $\mu$-algebra. In the former case, since $M$ is maximal we have that $L$ is quasi-abelian by Corollary 2.5 , which is a contradiction.

Clearly, (ii) implies (i).

As an immediate consequence of the above corollary and Corollary 3.5 we have

Corollary 4.6. Let $F$ be of characteristic $\neq 2,3$. Then every proper subalgebra of $a$ Lie algebra $L$ which is modular* must be quasi-abelian.

Proof. Let $U<L$ be modular* in $L$. Take a subalgebra $S$ of $L$ containing $U$ such that $U$ is maximal in $S$. If $S$ is solvable, then by Corollary 3.5 we have that $U$ is 
quasi-abelian. If $S$ is nonsolvable, then by Corollary 4.5 it follows that $S$ is a $\mu$-algebra and so $\operatorname{dim} U=1$. The proof is complete.

\section{Lie Algebras all of whose maximal subalgebras are modular*}

In this section the ground field is an arbitrary field.

Theorem 5.1. Let $L$ be a Lie algebra over any field. The following are equivalent:

(i) every maximal subalgebra of $L$ is modular* in $L$,

(ii) every subalgebra of $L$ is modular * in $L$,

(iii) every subalgebra of $L$ is modular in $L$,

(iv) the lattice $\mathcal{L}(L)$ of all subalgebras of $L$ is modular.

Proof. (i) implies (ii): By hypothesis, every maximal subalgebra of $L$ is modular* in $L$. By the height $\mathrm{h}(S)$ of a subalgebra $S$ of $L$, we mean the minimum length $h$ of chains

$$
S=M_{h}<M_{h-1}<\ldots<M_{1}<M_{0}=L
$$

such that $M_{i}$ is maximal in $M_{i-1}$ for every $1 \leq i \leq h$. If $\mathrm{h}(S)=1$, then $U$ is maximal in $L$. So $U$ is modular* in $L$. We proceed by induction on $h$. Then suppose that every subalgebra of $L$ having height less than or equal to $h-1$ is modular* in $L$ and let $S$ be a subalgebra of $L$ such that $\mathrm{h}(S)=h$. Pick $x \in M_{h-2}, x \notin M_{h-1}$. Put $B=((x)), C=S$ and $U=M_{h-1}$. We have $U \cap B=0$ and so $\langle U \cap B, C\rangle=C=S$. Also, we have $\mathbf{h}(U) \leq h-1$ and so $U$ is modular* in $L$, by the inductive hypothesis. By the second modular* identity, it follows that $\langle B, C\rangle \cap U=S$. Now take a maximal subalgebra $T$ of $M_{h-2}$ containing $\langle B, C\rangle$. We see that $h(T) \leq h-1$ and so $T$ is modular* in $L$. Moreover, we have $S \leq T \cap M_{h-1} \leq M_{h-1}$. Since $S$ is maximal in $M_{h-1}$, this yields either $S=T \cap M_{h-1}$ or $M_{h-1} \leq T \leq M_{h-2}$. In the former case, we find that $S$ is modular* in $L$ since so are $T$ and $M_{h-1}$ ([3, Lemma 2.3]). In the latter case, we have $T=M_{h-1}$ since $M_{h-1}$ is maximal in $M_{h-2}$ and $T \neq M_{h-2}$. But then we get $x \in T \leq M_{h-1}$, which is a contradiction.

(ii) implies (iii): Let $U \leq L$. To show that $U$ is modular in $L$, we only need to prove that the second modular identity for $U$ holds. To do that, take a subalgebra $C$ of $L$ containing $U$ and any subalgebra $B$ of $L$. By hypothesis, $C$ is modular* in $L$. By the second modular* identity for $C$, it follows

$$
\langle C \cap B, U\rangle=\langle B, U\rangle \cap C .
$$

This is just what we needed to prove.

(iii) implies (ii): It is the dual of (ii) implies (iii). Obviously, (iii) and (iv) are equivalent and (ii) implies (i). The proof is complete. 


\section{Lie Algebras all of whose minimal subalgebras are modular*}

We recall that in lattice $\mathcal{L}$ it said that $Y$ covers $X$ if $X<Y$ and moreover $X<Z<Y$ is not satisfied by any $Z$. A lattice $\mathcal{L}$ is called upper semimodular if $X$ covers $X \cap Y$ then $X \vee Y$ covers $Y$.

By Lemma 2.12 of [3], a minimal subalgebra $A$ of $L$ is modular* if and only if $S$ is maximal in $\langle S, A\rangle$ for every subalgebra $S$ of $L$ such that $A \cap S=0$. It follows that if every minimal subalgebra of $L$ is modular* the the lattice $\mathcal{L}(L)$ is upper semimodular, by a known result of lattice theory (see [10]).

When $\operatorname{char}(F) \neq 2,3$, the subalgebra lattice of a Lie algebra is upper semimodular if and only if it is modular (see [18]). For fields of characteristic three, the above result is not true in general. Indeed, the example given by Gein in [6, Example 2] shows a Lie algebra over a certain perfect field of characteristic three whose subalgebra lattice is upper semimodular but not modular.

Acknowledgements. The authors would like to thank the referee for his suggestions and comments.

\section{REFERENCES}

1. R. K. Amayo, Quasi-ideals of Lie algebras I, Proc. London Math. Soc. (3) 33, (1976), 28-36.

2. R. K. Amayo, and J. Schwarz, Modularity in Lie algebras, Hiroshima Math. J. 10 (1980), 311-332.

3. K. Bowman and D. A. Towers, Modularity conditions in Lie algebras, Hiroshima Math. J. 19 (1989), 333-346.

4. A. G. GeIn, Semimodular Lie algebras, Sibirsk. Mat. Z. 17 (1976), 243-248 (translated in Siberian Math. J. 17 (1976), 243-248). $3-15$.

5. A. G. GeIn, Projections of solvable Lie algebras,Ural Gos. Univ. Math. Zap. 10 (1976),

6. A. G. GEIN, Modular rule and relative complements in the lattice of subalgebras of a Lie algebra, Soviet Math. 31 (1987), 22-32; translated from Izv. Vyssh. Uchebn. Zaved. Mat. 83 (1987), 18-25.

7. A. G. Gern, On modular subalgebras of Lie algebras, Ural Gos. Univ. Mat. Zap. 14 (1987), 27-33.

8. B. Kolman, Semi-modular Lie algebras, J. Sci. Hiroshima Univ. Ser, A-I 29 (1965), 149-163.

9. A. LASHKI, On Lie algebras with modular lattices of subalgebras, J. Algebra 99 (1986), 80-86.

10. F. MAEDA and S. MAEDA, Theory of Symmetric Lattices (Springer-Verlag, Berlin, 1970).

11. D. A. Towers, Lie Algebras whose maximal subalgebras are modular*, Algebras, Groups Geom. 12 (1995), 89-98.

12. V. R. VAREA, Lie algebras none of whose Engel subalgebras are in intermediate position, Comm. Algebra 15 (1987), 2529-2543. 
13. V. R. VAREA, The subalgebra lattice of a supersolvable Lie algebra in Madison 1987 (Springer Lectures Notes in Math. 1373, Springer, New York 1989), 81-92.

14. V. R. VAREA, On modular subalgebras in Lie algebras of prime characteristic, Contemp. Math. 110 (1990), 289-307.

15. V. R. VAREA, Lie algebras having a modular subalgebra which is either a modular Lie algebra or simple of rank one, Contemp. Math. 131 (1992). 161-172.

16. V. R. VAREA, Modular subalgebras, quasi-ideals and inner ideals in Lie algebras of prime characteristic, Comm. Algebra 21 (1993), 4195-4218.

17. V. R. VAREA, Lie algebras whose proper subalgebras are either semisimple, abelian or almost-abelian, Hiroshima Math. J. 24 (1994), 221-241.

18. V. R. VArea, Upper semimodular and supersimple Lie algebras, Comm. Algebra, to appear.

Department of Mathematics and Statistics

UNIVERSITY OF CENTRAL LANCASHIRE

PRESTON

LANCASHIRE PR1 2HE

ENGLAND
DePartMent of MATHEMatics

UNIVERSITY OF ZARAGOZA

50009 ZARAGOZA

SPAIN 